\title{
VERIFICATION OF GOOD PRODUCTION PRACTICES THAT REDUCE THE RISK OF EXPOSURE OF PIGS TO TRICHINELLA
}

\author{
GAMBLE H.R.*, PYBURN D.**, ANDERSON L.A.*** \& MILLER L.E.***
}

\section{Summary :}

Control of Trichinella infection in swine has traditionally been accomplished by inspection of individual carcasses or by postslaughter processing to inactivate parasites. Recent declines in prevalence of this parasite in domestic swine, coupled with improvements in swine management systems, offer the opportunity to document pork safety during the production phase. We report here on a certification pilot study using an audit to document good production practices for swine relative to the risk of exposure to trichinae. Based on the results, improvements in the program have been made and further studies will be undertaken prior to launching a voluntary trichinae herd certification program in the United States.

KEY WORDS : swine, food safety, Trichinae, rodent control.

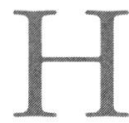
istorically, methods for assuring the safety of pork and pork products relative to Trichinella have included individual carcass inspection and processing methods such as cooking, freezing and curing. Among the industrialized countries, the United States is one of only a few that does not require some form of slaughter inspection for this parasite. The U.S. has relied on post-slaughter processing and consumer education regarding proper methods of meat preparation.

In those countries where routine slaughter inspection has been practiced for many years, Trichinella is rarely found in domestic pigs. In the U.S., Trichinella prevalence in domestic pigs has likewise declined to very low levels. In 1900, $2.5 \%$ of pigs tested in the U.S. were infected with trichinae (Gamble \& Bush, 1997). Prevalence declined to $0.95 \%$ in the 1930 's, $0.63 \%$ in $1952,0.16 \%$ in 1965 , and $0.12 \%$ in 1970 . The United States Department of Agriculture (USDA) National Animal Health Monitoring System's National Swine Survey in 1995 showed an infection rate of $0.013 \%$ (Gamble \& Bush, 1997). This decline in prevalence is primarily due to improvements in animal management systems

\footnotetext{
* U.S. Department of Agriculture, Agricultural Research Service.

** National Pork Producers Council.

*** U.S. Department of Agriculture, Animal and Plant Health Inspection Service.

Correspondence: H. Ray Gamble.

Tel.: 1-202-334-2787 - Fax: 1-202-334-2759 - email: rgamble@nas.edu
}

such as bio-secure housing systems and laws that prevent the feeding of raw garbage to swine.

Recently, there has been an interest in programs to assure the safety of pork relative to Trichinella by documenting management systems that reduce the risk of pig exposure to this parasite (Gamble et al., 2000; OIE International Animal Health Code, 1999; Knapen, 2000). These programs are based on a solid knowledge of risk factors associated with swine infection (Gamble et al., 1999). In the present study, we designed and pilot tested a system for auditing and certifying swine production systems that meet good production practices for reducing risk of exposure of pigs to Trichinella.

\section{MATERIALS AND METHODS}

\section{AUDIT OF GOOD PRODUCTION PRACTICES}

B ased on previous studies of risk factors associated with transmission of Trichinella to pigs (Gamble et al., 1999), an auditing document was constructed. The audit consisted of 56 questions focusing on elements of feed production and storage, building bio-security, rodent control, and general farm management and sanitation.

\section{TRAINING OF VETERINARIANS AS AUDITORS}

A one-day training program was developed to provide veterinary practitioners with the skills and knowledge to administer the audit for appropriate good production practices. Auditor training included basic knowledge on parasite biology and transmission, as well as information on bio-security, rodent control, and sanitary measures targeted at pork production facilities.

\section{AUDITING OF SWINE PRODUCTION FACILITIES}

A herd certification pilot was conducted in three states in the Midwestern U.S. (Iowa, Minnesota, South Dakota). The audit for good production practices was administered by trained, USDA accredited, veterinary practitioners on 353 swine farms in the three-state area. Results 
of audits were compiled and determinations made of which sites met all criteria for herd certification.

\section{TESTING OF PIGS FROM AUDITED PRODUCTION SITES}

All pigs raised on sites where audits were conducted were slaughtered at a single packing plant. A 1-gram sample from each carcass was tested by pooled diaphragm digestion (Gamble, 1998) and an enzymelinked immunosorbent assay (ELISA) (Safe-Path Laboratories, Minneapolis, MN, USA) was used to test serum from each pig.

\section{RESULTS}

A total of 34 veterinarians were trained as auditors for the trichinae herd certification pilot. These practitioners completed 198 audits on a total of 353 production sites (producers with multiple production sites completed a single audit). Of the sites surveyed, $59 \%$ were farrow-to-finish and $41 \%$ were grow-finish

Less than $4 \%$ of production sites included in this study met all criteria established within the audit for reduced risk management practices. Risks noted on the pork production sites are summarized in Table I. Most of the deficiencies were noted in the lack of a regular rodent control program around swine housing facilities. It was estimated that greater than $85 \%$ of these sites could meet the relevant good production practice criteria with minor improvements in management.

\begin{tabular}{lc}
\hline \multicolumn{1}{c}{ Risk } & $\begin{array}{c}\text { Percent farms } \\
\text { not meeting } \\
\text { requirements }\end{array}$ \\
\hline Proper rodent control program & $96 \%$ \\
Active rodent control (rats or mice and rats) & $81 \%$ \\
Feeding of household waste & $74 \%$ \\
Harborage near swine housing & $71 \%$ \\
Quality assurances for purchased feed & $70 \%$ \\
Proper rodent control in feed storage areas & $68 \%$ \\
Low rodent exclusion rating & $47 \%$ \\
Feed storage areas accessible to wildlife & $40 \%$ \\
Active rodent infestation (rats or mice and rats) & $37 \%$ \\
No perimeter around swine buildings & $35 \%$ \\
Ineffective sanitation practices & $12 \%$ \\
Evidence of cannibalism & $8 \%$ \\
\hline
\end{tabular}

Table I. - Risk factors for trichinae recorded during pork production site audits

To test seasonal variation in audit findings, a second questionnaire was administered on the same production sites at times ranging from four months to 14 months from the first audit. A total of 151 responses were obtained. Only minor variations in responses were obtained which could be attributed to seasonal variation. These variations included increased evidence of wild mammals in the vicinity of swine housing facilities during the spring season $(\mathrm{p}=0.01)$ and increased efforts on the part of producers to use rodent control methods inside swine housing facilities, also during the spring season $(\mathrm{p}=0.03)$.

From a total of 221,123 carcasses tested from audited farms during a six-month period, no Trichinella posi-
Records and Documentation
Animal movement records
Animal disposal plan
Feed information
Rodent control records
Records of animal movement are complete and up-to-date
A written plan and records of disposal of dead animals
A rodent control log is maintained for feed produced on-site
Quality assurances are maintained for purchased feed
If meat waste is fed, a waste-cooking log is maintained
Site Evaluation
Feed production and storage
Records of a rodent control system are complete and up-to-date
Swine housing
Site hygiene
Feed/feed ingredients are stored so they are inaccessible to rodents/wildlife Rodent control practices are in place
There is no evidence of active rat infestation
There is no evidence of active wildlife infestation
Feed spills are cleaned daily
There is no rodent harborage within 100 feet of the swine facility
There is a suitable "sterile zone" around the swine facility
There is rodent baiting in all areas of non-removable harborage
There is rodent baiting around building perimeter, entry, exit, load-out
The buildings are secure from entry by wildlife
Harborage for rodents is minimal inside swine buildings
Rodent baiting is in place at regular intervals inside buildings
General sanitation is good
There is an acceptable facility refuse storage and removal method
Disposal of dead pigs is consistent with animal disposal plan

Table II. - Elements of the good production practice audit document for herd certification. 
tives were detected by diaphragm digestion or by ELISA. No false-positive reactions were obtained using the ELISA, as compared with pooled digestion.

\section{DISCUSSION}

E ven with evidence that trichinae infection is becoming rare in the U.S., the perception of safety concerns about pork still exists with some consumers. The lack of a national testing program or an on-farm program to address trichinae may also be an impediment to the U.S. pork industry reaching its full market potential internationally.

In this study, we found that despite the relative rare occurrence of Trichinella infection in U.S. pigs, most pork production sites have some degree of risk for potential exposure from known sources of infection. These sources include rodents, wildlife, meat waste, and other swine carcasses. Thus, educational efforts are needed to provide pork producers with sufficient knowledge of appropriate good production practices that substantially reduce the risk of exposure of pigs to Trichinella. The most common deficiency noted in this study was in proper rodent control. Based on this observation, extensive efforts have been undertaken to educate producers and veterinarians servicing the pork industry on appropriate rodent control measures.

Based on the outcome of the study reported here, an improved, more succinct audit was developed (Table II) with objective measures of good production practices that should reduce or eliminate risk of exposure of pigs to sources of Trichinella. The new version of the audit is currently being used in large-scale pilots that will lead to the implementation of a voluntary herd certification program in the U.S. The proposed certification process includes the following elements: 1) veterinarians, trained in relevant good production practices, relative to trichinae, work with producers to ensure that risk factors are minimized on their farms; 2) the on-farm audit is used to document the reduction of trichinae infection risks; audits are done periodically to ensure that good production practices relative to trichinae remain in place; 3) a statistically valid sample of the national trichinae certified herd is tested at slaughter using diaphragm digestion or ELISA to verify the absence of infection; and 4) USDA veterinarians conduct random "spot audits" of certified production sites to ensure completeness and the integrity of the program.

\section{REFERENCES}

Gamble H.R. Trichinellosis. In OIE Manual of Standards for Diagnostic Tests and Vaccines, Chapter 3.5.3, 1998, pp. $477-480$.
Gamble H.R. \& Bush E. Seroprevalence of Trichinella infection in domestic swine based on the National Animal Health Monitoring System's 1990 and 1995 Swine surveys. Veterinary Parasitology, 1998, 80, 303-310.

Gamble H.R., Bessonov A.S., Cuperlovic K., Gajadhar A.A., Knapen F. van Noeckler K., Schenone H. \& Zhu X. International Commission on Trichinellosis: Recommendations on methods for the control of Trichinella in domestic and wild animals intended for human consumption. Veterinary Parasitology, 2000, 93, 393-408.

Gamble H.R., Brady R.C., Bulaga L.L., Berthoud,C.L., Smith W.G., Detweiler L.A., Miller L.E. \& Lautner E.A. Prevalence and risk factors for trichinellosis in domestic pigs in the northeastern United States. Veterinary Parasitology, 1999, $82,59-69$.

KNAPEN F. VAN. Control of trichinellosis by inspection and farm management practices. Veterinary Parasitology, 2000, 93, 385-392.

OIE International Animal Health Code, 8th Edition, Office Internationale des Epizooties, Paris, 1999, pp. 236-237. 